\title{
An outbreak of Salmonella Typhimurium traced back to salami, Denmark, April to June 2010
}

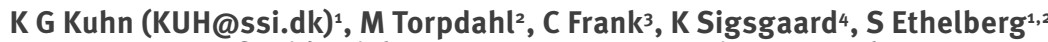

1. Department of Epidemiology, Statens Serum Institut, Copenhagen, Denmark

2. Department of Microbiology Surveillance and Research, Statens Serum Institut, Copenhagen, Denmark

3. Robert Koch Institut, Berlin, Germany

4. Danish Veterinary and Food Administration, Copenhagen, Denmark

Between April and June 2010, a small national outbreak of Salmonella Typhimurium with a particular multilocus variable-number tandem repeat analysis (MLVA) type was identified in Denmark through laboratory-based surveillance. The outbreak involved twenty cases, primarily living within the greater Copenhagen area. Half of the cases were children aged ten years or younger and 12 were male; three cases were hospitalised. A matched case-control study showed a strong link between illness and eating a particular salami product containing pork and venison, matched odds ratio (mOR):150, confidence interval (Cl): 19-1,600. The salami had been produced in Germany. Microbiological confirmation in food samples was sought but not obtained. Danish consumers were notified that they should return or dispose of any packages from the suspected salami batch. Because the salami product had potentially been sold in other European countries, the European Centre for Disease Prevention and Control urgent enquiry and Rapid Alert System for Food and Feed systems were used to highlight the possibility of outbreaks in these countries. Case-control studies are a strong tool in some outbreak investigations and evidence from such studies may give sufficient information to recall a food product.

\section{Introduction}

Salmonella enterica is the second most common cause of bacterial gastroenteritis in Denmark. A series of national control and intervention programmes have reduced the annual incidence to less than 30 cases per 100,000 population in 2010 [1] compared to 96 in 1997 [2]. However, imported products, including products from other European Union (EU) Member States, are not monitored within the Danish national programmes, but are rather tested for Salmonella by random sampling at import and, less often, during retail product control. Ready-to-eat products sold in Denmark are required to be free of Salmonella.

The circulation of Salmonella serovars in humans and animals in Denmark is monitored by the mandatory national human laboratory surveillance system and by analysing data derived from isolates from animals and food items of animal origin as part of the control programmes. In 2010, 33\% (521) of all registered human cases in Denmark were caused by $S$. Typhimurium [1]. Human isolates are sent from clinical laboratories to Statens Serum Institut (SSI) for typing, which for $S$. Typhimurium isolates include multilocus variable-number tandem repeat analysis (MLVA). MLVA is a typing method which has been shown to have good discriminatory power within $S$. Typhimurium [3]. Clusters of $S$. Typhimurium patientisolates with identical MLVA profiles are treated as potential outbreaks. Routine MLVA typing is now standard practise for surveillance of human $S$. Typhimurium infections in Denmark. This allows the detection of outbreaks that would otherwise have remained undiscovered.

On 15 April 2010, a cluster of 11 cases with identical S. Typhimurium MLVA profiles was detected, with all cases notified in April. Cases were invited to respond to a hypothesis-generating questionnaire by telephone. Two affected families independently indicated having consumed a certain unusual type of salami, which led to a working hypothesis that consumption of this salami product was associated with infection with $S$. Typhimurium. Here we describe the investigations undertaken to confirm this hypothesis, identify the source of the outbreak and to trace-back the suspected product.

\section{Methods}

MLVA was performed using primers described by Lindstedt et al [4] in the widely accepted method for $S$. Typhimurium. Of the five loci, STTR9 and STTR6 were labelled with 6-FAM, STTR5 and STTR3 with HEX and STTR with NED [5]. The primers were used in a single multiplex PCR followed by detection on an $A B I_{310}$ [6].

Phage typing was undertaken following the Anderson typing scheme [7]. 
Case definition and case-control study

For this investigation, a case was defined as a person residing in Denmark, who became ill with symptoms of gastroenteritis (diarrhoea and/or vomiting) after 1 April 2010, whose culture results yielded the outbreak strain and who had not travelled abroad between 25 March and 14 June. The outbreak strain was defined as $S$. Typhimurium having MLVA profile 3-14-12-NA-211.

Following initial hypothesis-generating patient interviews using a standard $S$. Typhimurium trawling questionnaire, a case-control study was initiated on 14 June, immediately after the discovery that a salami product may have been the source of infection. Controls were selected from the Danish population registry [8], matched by municipality, sex, and date of birth. To create a more robust statistical analysis, three controls were identified and interviewed for each case. Participants were interviewed by phone using a tailored questionnaire focusing on consumption of various types of meats, cold cuts, places where food was bought, as well as other exposures. Controls who experienced symptoms of gastroenteritis (diarrhoea and/or vomiting) or who had travelled outside Denmark during April and May 2010 were excluded.

\section{Statistical analysis}

Data from case and control questionnaires were entered into an EpiData database [9]. Statistical analyses were conducted in STATA 10 (StataCorp, TX). In order to examine relationships between each exposure and disease, odds ratios (ORs), matched odds ratios (mORs) and $95 \%$ confidence intervals $(\mathrm{CI})$ were calculated.

\section{International aspects}

On 15 June, an urgent enquiry was published through the European Centre for Disease Prevention and Control (ECDC) Epidemic Intelligence Information System (EPIS) and on 16 June a Rapid Alert System for Food and Feed (RASFF) notification was issued. The RASFF notification led to inspection by the German food authorities of the factory where the salami was produced.

\section{FIGURE}

Cases of Salmonella Typhimurium with the outbreak MLVA type, by week of disease onset, Denmark, 5 April-6 June $2010(n=20)$

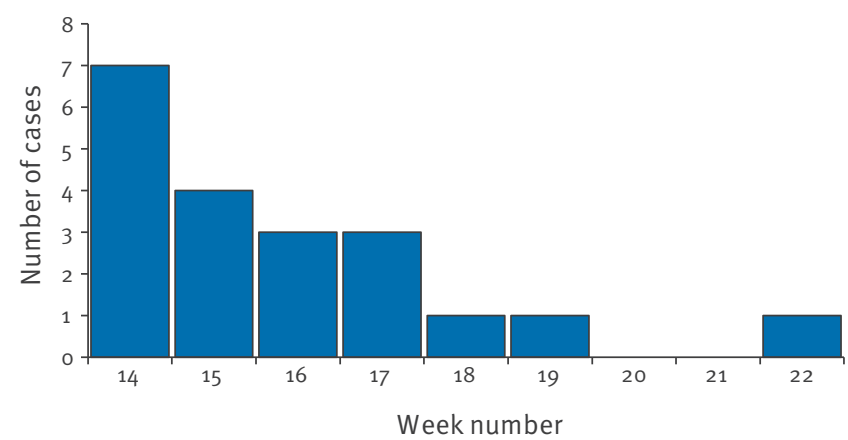

\section{Results}

During the time of the outbreak, 5 April to 6 June, 20 patient isolates (one isolate per patient) with the specific MLVA type were found over a period of nine weeks (Figure).

The median age of the patients was 20 years (range 1-69). Half of the cases (10) were children aged 11 years or younger and twelve were male. Three cases were hospitalised from symptoms caused by the infection, but no deaths occurred. The majority of patients (12 of 20) lived within the greater Copenhagen area which covers about $34 \%$ of the Danish population of approximately $5 \cdot 5$ million.

Trawling interviews using a generic Salmonella outbreak questionnaire led to a hypothesis concerning salami, when the majority of interviewed cases reported using the same supermarket chain when buying food and two families with cases mentioned having bought the same specific type of salami in that chain. The salami in question contained venison (meat from deer) and was traced back to a German producer which manufactures ready-to-eat products for the abovementioned Danish supermarket chain. Considering the disease onset dates and the shelf life of the salami (one and a half months), it was determined that the potentially contaminated packages of salami were from a single batch labelled with a use-by-date between 6 April and 16 June 2010.

For the case-control study, 17 of the 20 identified cases and 79 controls were interviewed. A total of 16 controls were excluded from the analysis; five due to symptoms of gastrointestinal illness and 11 due to having travelled abroad. Consuming Brand $\mathrm{X}$ salami with smoked venison and pork was strongly associated with illness (Table). All cases but two reported having consumed this salami during the week before illness onset ( $m O R$ : 150; 95\% Cl: 19-1,600; P<0.0001). Illness was also significantly associated with consumption of salami

\section{TABLE}

Results of single-variate analysis of selected exposures from case-control study, Denmark $2010(n=80)$

\begin{tabular}{|l|c|c|c|}
\hline Exposure & $\begin{array}{c}\text { Cases } \\
\mathrm{n} / \mathrm{N}\end{array}$ & $\begin{array}{c}\text { Controls } \\
\mathrm{n} / \mathrm{N}\end{array}$ & $\begin{array}{c}\text { Matched Odds } \\
\text { Ratio }(95 \% \mathrm{Cl})\end{array}$ \\
\hline Pork & $2 / 17$ & $29 / 63$ & $0.2(0.03-0.7)$ \\
\hline Chicken & $8 / 17$ & $39 / 63$ & $0.5(0.2-1.6)$ \\
\hline Turkey & $2 / 17$ & $8 / 63$ & $1.3(0.2-6.1)$ \\
\hline Beef & $7 / 17$ & $29 / 63$ & $2.0(0.2-95.8)$ \\
\hline Sliced rolled meat & $2 / 17$ & $4 / 63$ & $1.1(0.3-4.1)$ \\
\hline Salami & $15 / 17$ & $34 / 63$ & $6.4(1.3-61.1)$ \\
\hline Beef salami & $4 / 17$ & $17 / 63$ & $0.8(0.2-3.2)$ \\
\hline Game salami & $14 / 17$ & $3 / 63$ & $93(13.9-723.9)$ \\
\hline $\begin{array}{l}\text { Brand X smoked deer } \\
\text { and pork salami }\end{array}$ & $15 / 17$ & $3 / 63$ & $150(18.8-1626)$ \\
\hline
\end{tabular}

Multiple exposures to food items were possible. 
in general (mOR: $6.4 ; 95 \% \mathrm{Cl}: 1.3-61)$ and answering yes to having consumed salami containing game meat (mOR: 93; 95\% Cl: 14-720) (Table).

The outbreak strain was initially phage typed to be a mixture of the biphasic S. Typhimurium DT120 and DT7 with resistance to ampicillin, streptomycin, sulphamethoxazole and tetracycline. Separate phage typing of several isolates at the World Health Organisation Collaborative Centre for phage typing of Salmonella (Health Protection Agency, Colindale, United Kingdom [10]), later confirmed that the type was actually DT193 with several non-specific reactions. These results were obtained after the EPIS and RASFF notifications had been issued. In contrast, the MLVA type was highly characteristic and non-varied and thus the case definition was purely based on the MLVA results. All patient isolates in the outbreak had the same MLVA type.

At the time of the discovery, the salami batch, which had most likely caused the outbreak, had passed its expiry date and therefore no recalls were made. Additionally, it was not possible for the regional food authorities to obtain any salami for microbiological examination and thus no samples of salami from the suspected batch were available for microbiological testing. The inspection by German authorities of the factory where the salami was produced did not identify problems in the factory nor a contamination of the meat in question. The Danish supermarket chain selling the salami also operates in other European countries, however investigation by the Danish Veterinary and Food Administration indicated that the particular salami from the same producer had only been sold in Denmark and Germany. There was no indication that the implicated batch of salami had also been sold in Germany, although similar products from the same producer were available in German stores of the same supermarket chain. The batch-focused food trace back yielded no information if and how the deer meat salami sold in German stores was related to the Danish batch.

Danish food authorities issued a warning for consumers to return or dispose of any packages of Brand $X$ smoked deer and pork salami with a use-by-date between 6 April and 16 June 2010.

\section{Discussion}

A small national outbreak of S. Typhimurium DT193 was identified through laboratory-based surveillance using MLVA typing. The most likely conclusion, based on the findings of the case-control study, disease onset dates as well as product supply and distribution, was that the outbreak was caused by specific sliced salami containing smoked deer and pork meat. When the suspected outbreak source was detected in June, new cases had not been identified for several weeks. It was concluded that a single batch of infected salami had caused the outbreak. Survival of Salmonella in readyto-eat products has the potential to cause illness and salami has on several occasions been identified as the food vehicle for $S$. Typhimurium [11-13]. A recent multistate outbreak of S. Montevideo in the United States was shown to have been caused by salami products containing contaminated red and black pepper, additionally highlighting the importance of post-processing contamination of ready-to-eat products [14].

Following the EPIS urgent enquiry, Germany reviewed the $S$. Typhimurium situation during April and May 2010. MLVA typing is not routinely performed in Germany; thus it would only be possible to detect a corresponding outbreak by looking at $S$. Typhimurium case numbers in general. During the time in question, a similar number of cases of $S$. Typhimurium were notified from Germany as a whole compared to April and May in previous years $(1,479$ vs a mean of 1,489 in 2007-9). However, restricting the comparison to only those six federal states where stores from the implicated Danish supermarket chain are widespread, a $24 \%$ increase in $S$. Typhimurium cases was observed, compared to the mean of 2007-9 (645 vs 519 cases).This excess was mainly in adult males above 18 years old and strongest between 12 and 18 April. Due to the time elapsed, these cases were not investigated further.

The observed regional increase in German $S$. Typhimurium cases, restricted to states with branches of the Danish supermarket chain, is suggestive of a link to the Danish outbreak. Differences in age distribution between the German and the Danish cases may be explained by the fact that food preferences for such an unusual sausage product may vary geographically. Without MLVA confirmation, we cannot be sure that the German regional increase was due to the same type of S. Typhimurium.

No further cases matching the outbreak profile were reported from Europe.

The investigations described in this paper highlight the usefulness of typing methods in combination with simple case-control studies to detect an outbreak and identify possible sources of infection. Although no samples were available for testing, it was concluded that the smoked pork and deer meat salami was the likely source of infection in this outbreak, solely based on the case-control investigation results. Vehicle identification was aided by the unusual type of salami, which made it stand out in the memories of the patients.

The international dimension of this outbreak in Denmark is not only demonstrated by the fact that the salami at the source was produced in Germany, but also because the Danish supermarket chain selling the salami operates in other European countries, including Germany and England. Therefore the ECDC urgent enquiry and the RASSF systems were used to highlight the potential for similar outbreaks in these countries. It is not unlikely that the particular salami had caused cases of salmonellosis in countries other than Denmark, however, the use of different national subtyping systems 
made it difficult to detect such cases by the Danish definition in these countries. This is potentially a serious problem in a multi-national outbreak situation. To rapidly determine if disease outbreaks in several countries are caused by the same strain, methods for molecular typing should be standardised throughout the EU. Ammon and Tauxe [15] highlight the need for developing a consensus about which methods to use, their application in all laboratories as well as implementing additional methods, such as resistance testing and pulsed-field gel electrophoresis, in particular situations. Another potential challenge within the EU is the investigation of a food outbreak source by microbiology and trace-back in situations where the food is produced in a different country from the one where the outbreak takes place. Encouragingly, however, in this outbreak a food investigation was made by German authorities as a result of an RASSF notification from another country, based purely on the results of a casecontrol study, even though there were no confirmed cases in Germany.

\section{Acknowledgements}

The authors are grateful to Gitte Sørensen at FOOD, Danish Technical University (DTU) for phage typing of isolates and general information about phage typing. We would also like to thank clinical laboratories in Denmark for supplying us with patient isolates.

\section{References}

1. Ethelberg S, Mølbak K, Olsen KEP, Nielsen EM, Scheutz FM. Zoonotic gastroenteric infections 2010. EpiNews. 2011;12.

2. Anonymous 2006. Annual report on zoonoses in Denmark 2005. Ministry of Family and Consumer Affairs, Copenhagen, Denmark.

3. Torpdahl M, Sørensen G, Lindstedt BA, Nielsen EM. Tandem repeat analysis for surveillance of human Salmonella Typhimurium infections. Emerg Infect Dis 2007;13(3):388-95.

4. Lindstedt BA, Vardund T, Aas L, Kapperud G. Multiple-locus variable-number tandem-repeat analysis of Salmonella enterica subsp enterica serovar Typhimurium using PCR multiplexing and multicolour capillary electrophoresis. J Microbiol Methods 2004;59 (2):163-72.

5. Larsson JT, Torpdahl M, Petersen RF, Sørensen G, Lindstedt BA, Nielsen EM. Development of a new nomenclature for Salmonella Typhimurium multilocus variable number of tandem repeats analysis (MLVA). Euro Surveill. 2009;14(15):pii=19174. Available from: http://www.eurosurveillance.org/ViewArticle. aspx?Articleld $=19174$

6. Torpdahl M, Sørensen G, Ethelberg S, Sandø G, Kammegard $\mathrm{K}$, Jannok Porsbo L. A regional outbreak of S. Typhimurium in Denmark and identification of the source using MLVA tuping. Euro Surveill. 2006;10(45):pii 2829. Available from: http:// www.eurosurveillance.org/ViewArticle.aspx?Articleld $=621$

7. Anderson ES, Ward LR, de Saxe MJ, de Sa JD. Bacteriophagetyping designations of Salmonella typhimurium. J Hyg. 1977;78(2):297-300.

8. Pedersen CB, Gøtzsche H, Møller JO, Mortensen PB. The Danish Civil Registration System. A cohort of eight million. Dan Med Bull. 2006;53(4):441-9.

9. Lauritsen JM. (Ed.) EpiData Data Entry, Data Management and basic Statistical Analysis System. Odense Denmark, EpiData Association, 2000-2008. Available from: http://www.epidata. $\mathrm{dk} /$

10. Baggesen DL, Sørensen G, Nielsen EM, Wegener HC. Phage typing of Salmonella Typhimurium - is it still a useful tool for surveillance and outbreak investigation? Euro Surveill. 2010;15(4):pii=19471. Available from: http://www. eurosurveillance.org/ViewArticle.aspx?Articleld=19471
11. Cowden JM, O'Mahony M, Bartlett CL, Rana B, Smyth B, Lynch $D$ et al. A national outbreak of Salmonella typhimurium DT124 caused by contaminated salami sticks. Epidemiol Infect. 1989;103(2):219-25.

12. Hjertqvist M, Luzzi I, Löfdahl S, Olsson A, Rådal J, Andersson Y. Unusual phage pattern of Salmonella Typhimurium isolated from Swedish patients and Italian salami. Euro Surveill 2006;11(6): pii=2896. Available from: http://www. eurosurveillance.org/ViewArticle.aspx?Articleld=2896

13. Luzzi I, Galetta P, Mazzari M, Rizzo C, Dionisi AM, Filetici E et al. An Easter outbreak of Salmonella Typhimurium DT104A associated with traditional pork salami in Italy. Euro Surveill 2007;12(4):pii=702. Available from: http://www. eurosurveillance.org/ViewArticle.aspx?Articleld =702

14. Centers for Disease Control and Prevention (CDC). Salmonella Montevideo infections associated with salami products made with contaminated imported black and red pepper - United States July 2009 - April 2010. MMWR Morb Mortal Wkly Rep. 2010;59(50):1647-50.

15. Ammon A, Tauxe RV. Investigation of multi-national foodborne outbreaks in Europe: some challenges remain. Epidemiol Infect 2007;135(6):887-9. 